\title{
ESTRESSE OCUPACIONAL: INFLUÊNCIA DAS MUDANÇAS GERADAS NA ÁREA DE TECNOLOGIA DURANTE IMPLANTAÇÃO DO SAP R/3 $3^{1}$ *
}

\author{
OCUPATIONAL STRESS: INFLUENCE OF THE CHANGES IN THE \\ TECHNOLOGY DEPARTMENT DURING THE SAP R/3 IMPLEMENTATION
}

\author{
Daniella Forster ${ }^{2}$ \\ Wesley Vieira da Silva ${ }^{3}$ \\ Karina De Dea Roglio ${ }^{4}$ \\ Luiz Carlos Duclós ${ }^{5}$
}

\begin{abstract}
RESUMO: A pesquisa relatada neste artigo tem como objetivo analisar os principais fatores causadores de estresse entre profissionais da área de tecnologia da informação durante um processo de adoção do sistema SAP $\mathrm{R} / 3$. Com esse objetivo, foi realizado um estudo de caso em uma empresa multinacional instalada em Curitiba. Aplicou-se um questionário estruturado, construído com base na versão resumida da "job stress scale" e adaptada para o português por Alves et al. (2004). Questões foram incluídas ao modelo, com o objetivo de analisar aspectos pertinentes ao momento específico da organização, mantendo-se a relação com o modelo proposto. Para a análise dos dados, foram utilizados dois métodos de pesquisa complementares: regressão logística multinominal e análise fatorial. A primeira métrica permitiu avaliar que as características dos grupos chamados "Demanda", "Controle" e "Apoio Social" estavam adequadas às funções. A segunda revelou a existência de sete fatores, que tiveram a presença de 22 das 38 variáveis listadas. Os resultados indicaram a presença de estresse a partir dos sete fatores sobressalentes e da alta frequência da combinação: forte exigência, baixo controle e pouco apoio social. Em ambas as situações houve compatibilidade com o modelo de ExigênciaControle-Suporte utilizado.
\end{abstract}

PALAVRAS-CHAVE: Estresse Ocupacional. Modelo de Exigência-Controle-Suporte. Sistema SAP.

ABSTRACT: The research presented in this paper aims to find out the main stressors from 59 professionals working in the technology department of a multinational company in Curitiba, which was implementing the SAP R/3. We applied a structured questionnaire adapted from the "job stress scale", which is based on the demand-contro-social supportl model of Karasek and Theorell (1990). Other issues were included in order to examine some relevant aspects to the organizational specific context, although preserving the relationship with the model proposed. Data were analysed with two complementary methods: multinomial logistic regression and factorial analysis. In the first metric, we verified if the characteristics of "Demand", "Control" and "Social Support" were appropriate to the task. The second showed the existence of seven factors, which had the presence of 22 of the 38 variables investigated. The results indicated the presence of stress in seven factors and the high frequency of the combination: strong demand, low control and low social support. In both situations, data matched the demand-control-social support model.

KEYWORDS: Stress, Demand-Control-Social Support ModeL, SAP System.

\footnotetext{
${ }^{1}$ Artigo Recebido em 27.02.2009. Revisado por pares em 08.02.2010. Recomendado em 18.08.2010 por Leomar dos Santos Editor. Publicado em 04.04.2011.

Organização Responsável pelo periódico: Universidade regional de Blumenau - FURB - www.furb.br/rn
}

\footnotetext{
* Versão revisada do artigo apresentado no I ENADI (Encontro de Administração da Informação) em 26 de outubro de 2007.

${ }^{2}$ Pontifícia Universidade Católica do Paraná - PUCPR - daniellaforster@,forsterconsult.com

${ }^{3}$ Pontifícia Universidade Católica do Paraná - PUCPR - wesley.vieira@pucpr.br

${ }^{4}$ Universidade Federal do Paraná - UFPR - karinaroglio@gmail.com

${ }^{5}$ Pontifícia Universidade Católica do Paraná - PUCPR - luiz.duclos@pucpr.br
} 


\section{INTRODUÇÃO}

O estresse no trabalho é um fenômeno reconhecido em diversas pesquisas, que se expressa de diferentes formas e influencia os trabalhadores também de diferentes maneiras, de acordo com especificidades do contexto organizacional e características individuais. Seu estudo em diferentes contextos contribui para aprofundar a compreensão desse fenômeno e a identificação de possibilidades para minimizar seus efeitos negativos sobre a produtividade, a satisfação e o comprometimento dos trabalhadores (MICHAEL; COURT; PETAL, 2009). Os resultados da pesquisa ora relatada podem auxiliar as organizações a compreender o estresse gerado em situações de mudança e a gerenciar as suas implicações de modo mais efetivo.

A área de tecnologia da informação é reconhecida, sobretudo nas empresas de grande porte, como essencial à estrutura organizacional. A informática atualmente não está restrita ao cadastro e controle, mas se estende aos processos que permitem uma integração efetiva entre as diversas áreas, a percepção da organização como um todo e a sua inserção no mundo globalizado. Estas constatações são claramente observadas e compreendidas a partir da década de 90, pela crescente implantação de sistemas ERP (Enterprise Resource Planning), mais especificamente do sistema SAP.

Albertin (2001) expõe que as organizações buscam cada vez mais o uso intensivo de Tecnologia de Informação (TI) como uma poderosa ferramenta, que altera as bases competitivas, estratégicas e operacionais das empresas. Desenvolver e implementar as aplicações de TI em uma organização apresenta suas próprias características, as quais devem ser estudadas e planejadas de acordo com o ambiente. O autor ainda destaca que a TI tem sido utilizada em larga escala para interligar suas várias áreas, fornecedores e clientes, processar um número grande de transações e atender a uma quantidade de clientes de forma rápida, segura e, muitas vezes, personalizada. Portanto, existe a percepção de que a TI é uma área de extrema relevância em qualquer organização e, pela sua característica intrínseca de integrar processos e pessoas, gera níveis de complexidade que, em determinadas situações, podem gerar problemas que exigem uma gestão efetiva.

Gonçalves (2000) enfatiza que a maioria dos processos importantes das empresas atravessa as fronteiras das áreas funcionais, sendo conhecidos como processos interfuncionais ou interdepartamentais. $\mathrm{O}$ autor exemplifica a partir do impacto da tecnologia no trabalho individual e entre pessoas que atuam juntas em prol de determinada tarefa ou projeto. A tecnologia afeta diretamente a comunicação dentro e fora da organização, oferecendo alternativas eletrônicas, extremamente eficazes, mas que exigem novas posturas e, portanto, novas habilidades a serem adquiridas. É irrefutável que um projeto que envolva a tecnologia afeta diretamente o comportamento das pessoas, a partir do momento que apresenta novas demandas e atitudes. Os processos de implementação de sistemas ERP-SAP estão inseridos nesse contexto e é comum agravarem os níveis de estresse das pessoas envolvidas.

No Brasil, existem muitos estudos sobre estresse, principalmente relacionados à área de saúde. Sobre a área de TI, especificamente, Rocha e Debert-Ribeiro (2001) realizaram um estudo comparativo acerca do trabalho, saúde e gênero entre analistas de sistemas. A conclusão das autoras sugere repercussões na saúde dos analistas associadas às exigências no 


\section{ESTRESSE OCUPACIONAL: INFLUÊNCIA DAS MUDANÇAS GERADAS NA ÁREA DE TECNOLOGIA DURANTE IMPLANTAÇÃO DO SAP R/3}

trabalho e ao papel da mulher na sociedade. Outro estudo foi publicado na revista especializada em Tecnologia de Informação: Computerworld. Realizado pela SWNS, consultoria norte-americana, os resultados mostraram que 97\% dos profissionais da área de tecnologia em todo o mundo relatam estresse diário em seu ambiente de trabalho, à frente de outras profissões. As principais fontes de estresse foram identificadas como dificuldades em cumprir prazos para suas tarefas e estresse ao assumir tarefas conduzidas por outros profissionais.

A presente pesquisa tem como objetivo analisar os principais fatores causadores de estresse entre profissionais da área de tecnologia da informação durante um processo de adoção do sistema SAP R/3. Com esse objetivo, foi realizado um estudo de caso em uma empresa multinacional instalada em Curitiba. Na empresa investigada, identificou-se que a implantação do sistema SAP R/3 gerou demissões, novas contratações, além de muitas alterações nos procedimentos e tecnologias propriamente ditas. Como conseqüência, a Diretoria Regional da empresa no Paraná observou a área de TI como sujeita ao maior nível de estresse na organização e solicitou um estudo para identificar as principais causas do mesmo.

Para atender ao objetivo proposto, nas duas seções seguintes é apresentada uma revisão de literatura, que trata do estresse no contexto organizacional e do modelo de levantamento de estresse de Karasek e Theorell (1990). Na seqüência, detalha-se a metodologia utilizada na pesquisa empírica. Em seguida, os dados obtidos são apresentados e analisados com base no referencial teórico adotado na pesquisa. Por fim, são desenvolvidas as considerações finais e recomendações para a realização de pesquisas futuras.

\section{O ESTRESSE NO CONTEXTO ORGANIZACIONAL}

Lipp (1996) afirma que o termo stress foi introduzido por Selye em 1936 para denominar uma síndrome produzida por agentes nocivos, que era uma resposta não-específica a algumas situações em que o organismo se enfraquecia ou adoecia, pelo esforço dos processos fisiológicos para manter um estado de equilíbrio interno no organismo. O stress é um estado fundamental da existência, que acontece por meio de um stressor, que é agente que faz, de alguma forma, com que o indivíduo tenha uma reação que resulta em um estado de desequilíbrio e abrange as mudanças que acontecem no mundo interno ou externo, como por exemplo, as mudanças de emprego, de chefes, ou mesmo o divórcio ou nascimento de filhos. O stress acontece quando esses stressors em demasia acabam se acumulando e gerando uma série de reações (SELYE, 1956).

No século XIX, a medicina tratou o estresse como um fator de má condição de saúde. No século XX, mais especificamente na década de 30, Walter Cannon definiu o estresse como um distúrbio de homeostase, observável sob determinadas condições (frio, falta de oxigênio...). No final da década de 70 , cresceu a atenção para o estudo do impacto das mudanças sociais sobre a vida humana, além dos aspectos ambientais dos fenômenos psíquicos. Desde então, é crescente a preocupação das organizações com os efeitos negativos do estresse sobre os seus empregados. Richardson e Rothstein (2008, p. 69) afirmam que "o interesse por estratégias de redução do estresse no trabalho vem aumentando de forma rápida 
e contínua desde os anos 70". Os mesmos autores mencionam que "embora não seja possível eliminar totalmente o estresse, as pessoas podem aprender a gerenciá-lo. Muitas organizações adotaram programas de treinamento para reduzir os níveis de estresse da sua força de trabalho" (RICHARDSON; ROTHSTEIN, 2008, p. 69). Ongori e Agolla (2008) revelam que o estresse ocupacional afeta o turnover, a produtividade e o desempenho da organização. Gestores em várias organizações vivem o dilema sobre quais são as intervenções necessárias para minimizar os custos associados com o estresse ocupacional. Por isso, é essencial compreender as causas, sintomas e efeitos desse estresse sobre o desempenho organizacional.

Nesse contexto, diversos estudos abordam o tema estresse nas organizações. Richardson e Rothstein (2008) realizaram uma meta-análise com o objetivo de revisar e sintetizar estudos experimentais sobre intervenções de gestão do estresse (Stress Management Intervention - SMI) que foram conduzidos (e relatados) em diversas disciplinas (educação, saúde, estudos organizacionais e psicologia) para identificar o que funciona, o quão bem funciona, e onde e para quem funciona. A pesquisa desenvolvida por Michael, Court e Petal (2009) teve como propósito examinar o impacto do estresse no trabalho sobre o comprometimento organizacional. Randalla, Nielsenb e Tvedtc (2009) desenvolveram cinco escalas, denominadas Intervention Process Measure (IPM), para mensurar a avaliação dos empregados sobre intervenções direcionadas ao gerenciamento do nível de estresse organizacional. Ongori e Agolla (2008), cuja pesquisa teve como objetivo investigar o estresse ocupacional e os seus efeitos sobre o desempenho organizacional, confirmaram que os custos atrelados ao estresse ocupacional são altos e os seus impactos sobre os empregados não podem ser ignorados. A omissão da organização em gerenciar o estresse ocupacional pode deteriorar a rentabilidade organizacional por meio de litígios, morbidez e mortalidade.

O modelo de stress no trabalho, apresentado por Kanji-Chopra (Kanji-Chopra work stress model - KCWSM), é baseado em condições psicossociais do trabalho que precisam ser gerenciadas de modo adequado para não criar estresse no trabalho. "Essas condições incluem vários fatores psicossociais do trabalho reconhecíveis no indivíduo, como suporte, demanda de trabalho, controle do trabalho e ambiente de trabalho. A falta de funcionamento de um ou de todos esses fatores na organização indicam estresse no trabalho" (KANJI; CHOPRA, 2009, p. 568). Gilboa, Shirom, Fried e Cooper (2008) realizaram uma pesquisa quantitativa para investigar as relações entre sete fatores de estresse relacionados com o trabalho e o desempenho no trabalho (ambigüidade das tarefas, conflito de tarefas, sobrecarga de trabalho, insegurança no trabalho, conflito trabalho-família, incerteza ambiental e restrições situacionais). As pesquisas aqui mencionadas mostram a relevância que o tema estresse possui no contexto organizacional, sobretudo porque o aumento crescente da velocidade das transformações no ambiente de negócios gera mudanças constantes nas organizações e por conseguinte, um aumento dos níveis de estresse ocupacional, que comprometem os resultados organizacionais, a qualidade de vida dos indivíduos e a sustentabilidade humana e social.

\subsection{MODELO DE LEVANTAMENTO DE ESTRESSE}




\section{ESTRESSE OCUPACIONAL: INFLUÊNCIA DAS MUDANÇAS GERADAS NA ÁREA DE TECNOLOGIA DURANTE IMPLANTAÇÃO DO SAP R/3}

Na realização desta pesquisa, foi utilizado o modelo de Karasek e Theorell (1990). De acordo com França e Rodrigues (2002), diversos autores utilizam o modelo tridimensional de exigência-controle-suporte (Demand-Control-Support - DCS) de Karasek e Theorell (1990) para avaliar o nível de estresse no ambiente organizacional. Conti et al. (2006) analisaram as condições de trabalho como um aspecto negligenciado na implementação do sistema de produção Enxuta (Lean Production), especificamente as relações entre a implementação dessa tecnologia e o estresse dos empregados no trabalho. Neste estudo, o modelo de Karasek e Theorell (1990) foi utilizado para estabelecer conexões entre as práticas operacionais e o estresse no trabalho. Fox e Stallworth (2010) também utilizaram o modelo de demandacontrole-suporte de Karasek e Theorell (1990) como um dos referenciais para o desenvolvimento da estrutura modificada do modelo estressor-emoção-controle/suporte (modified stressor-emotion-control/support - SEC/S), que foi utilizado para mensurar os níveis de estresse causados por experiências de violência e de intimidação (bullying) em professores.

Em 1979, Karasek desenvolveu o Modelo de Exigência-Controle, onde a definição de estresse subjacente é atribuída à respostas físicas e emocionais prejudiciais. $\mathrm{O}$ autor conceitua as exigências como pressões de natureza psicológica, tanto quantitativas (rapidez e prazo na realização do trabalho) como qualitativas (conflitos entre demandas contraditórias). $\mathrm{O}$ controle se refere à possibilidade do trabalhador de utilizar suas habilidades intelectuais, bem como possuir autoridade para a realização de seu trabalho. Inicialmente, o autor propôs um modelo teórico bi-dimensional, considerando dois aspectos: a) as exigências do trabalho, que não estão em equilíbrio com as capacidades, recursos ou necessidades do profissional e b) a falta de autonomia para tomar decisões. Ao relacionar os níveis de exigência e controle, o modelo sugere diferentes situações de trabalho: a) alto desgaste: alta demanda e baixo controle; b) ativo: alta demanda e alto controle; c) passivo: baixa demanda e baixo controle; d) baixo desgaste: baixa demanda e alto controle (FRANÇA; RODRIGUES, 2002).

Posteriormente, Karasek e Theorell (1990) revisaram esse modelo, designando-o como Modelo de Exigência-Controle-Suporte (Demand-Control-Support - DCS), no qual incluíram o suporte ao lado do controle como moderador da relação estressor-esforço (stressor-strain relationship). O modelo incorporou os efeitos das exigências do trabalho (físicas e psicológicas), o controle e o suporte social (que inclui suporte físico e técnico). Trabalhos estressantes são associados com exigências altas, controle baixo e suporte social também baixo. Na nova versão do instrumento utilizado para avaliar os níveis de estresse, o número de questões foi reduzido de quarenta e nove para dezessete, assim distribuídas: cinco para avaliar a demanda, seis para avaliar o controle e seis para o apoio social. A adaptação dessa versão reduzida para a língua portuguesa foi tema da pesquisa intitulada "Versão Reduzida da 'Job Stress Scale': adaptação para o português", desenvolvida por Alves et al., 2004. O resultado dessa pesquisa confirma a validade e a confiabilidade da tradução.

Segundo França e Rodrigues (2002), o modelo de demanda-controle recebeu crítica referente ao foco dado às características do trabalho. Entretanto, os autores também citam o trabalho de Baker e Karasek, no qual argumentam que o modelo pode ser expandido para agregar outros fatores organizacionais. Os componentes do processo de estresse incluem: a) exigências de tempo, estrutura temporal do trabalho e ritmo; b) estrutura de tarefas; c) 
condições físicas; d) organização do trabalho; e) extra-organizacionais: fatores relacionados à insegurança no emprego e preocupações com a carreira; f) fontes externas ao trabalho: pessoais, família, relacionados à comunidade. Uma implicação interessante do modelo exposto é o fato de que intervenções na estrutura de decisão e na forma de organização do trabalho, que possibilitem maior flexibilidade e eqüidade, podem exercer grande influência nos níveis de tensão mental dos profissionais, comumente submetidos a altas exigências no que se refere aos resultados organizacionais (FRANÇA; RODRIGUES, 2002).

Para a operacionalização da presente pesquisa, foi utilizada a versão reduzida da $J o b$ Stress Scale (ALVES et al., 2004). Na definição do modelo de levantamento dos fatores de estresse, foram consideradas as principais características da área de TI que, em geral, está sujeita às pressões geradas pela exigência de entrega de trabalhos em prazos curtos, com os mesmos padrões de qualidade; constante atualização técnica; integração entre os profissionais de diferentes áreas, o que acarreta em transferência de responsabilidade em diferentes atividades. O questionário utilizado é apresentado no Anexo 1.

\section{METODOLOGIA}

Esta seção destina-se à descrição da metodologia empregada na pesquisa de campo, e está subdividida em quatro subseções: 1 . caracterização da pesquisa; 2. população e amostra; 3. coleta e tratamento dos dados; 4. métodos de análise dos dados.

\subsection{Caracterização da pesquisa}

O método de pesquisa empregado neste estudo foi o dedutivo, definido por Lakatos e Marconi (1991) como um método no qual a busca por soluções parte de teorias ou leis previamente aceitas. No que se refere à natureza da aplicação, caracteriza-se como uma pesquisa aplicada, pois utiliza-se de conhecimentos provenientes de pesquisas básicas bem como as tecnológicas existentes, visando promover a geração de novos produtos e processo. Produz, também, conhecimentos que são disseminados pelos pesquisadores diretamente em empresas ou por meio de congressos, feiras, seminários ou mesmo manuais técnicos. No que diz respeito aos objetivos da pesquisa, esta pode ser caracterizada como uma pesquisa descritiva, pois segundo Gil (2002), a pesquisa descritiva estabelece relações entre variáveis por meio da descrição das características de determinado fenômeno ou população. Uma pesquisa pode, ainda, ser caracterizada em função do tempo de coleta e aplicação. Neste caso, constitui-se em um estudo de corte transversal porque envolve medições em um determinado período do tempo.

Em relação aos procedimentos técnicos utilizados, a pesquisa é classificada como pesquisa com delineamento ex-post facto, pois se refere a experimentos realizados após a ocorrência dos fatos e, sendo assim, o pesquisador não tem controle sobre as variáveis analisadas. 


\subsection{População e amostra}

O tipo de amostragem utilizado nessa pesquisa foi o não probabilístico, mais especificamente a amostragem não probabilística intencional ou por julgamento, onde o pesquisador seleciona os membros da população de acordo com o seu julgamento. Esse tipo de amostragem é bastante útil quando se conhece as características da população, o que evita vieses em termos de representatividade.A população inicialmente pesquisada foi de 70 indivíduos (correspondentes ao total de empregados devidamente registrados em carteira ou terceirizados pela empresa investigada), vinculados à área de Tecnologia de Informação (TI). A amostra pesquisada, após a eliminação dos outliers, refere-se ao total de 47 empregados da área de TI que se dispôs a participar da referida pesquisa.

\subsection{Coleta e tratamento dos dados}

Para a coleta de dados, elaborou-se um questionário estruturado contendo trinta e oito perguntas. Sua divisão originou quatro grupos a serem pesquisados: "Dados Pessoais", "Demanda", "Controle" e "Apoio Social". O primeiro grupo foi formado por dez perguntas sobre sexo, idade, estado civil, número de filhos, residência, grau de instrução, tempo de vínculo empregatício, cargo, participação do programa SAP e o rendimento familiar. Os grupos seguintes foram formados por perguntas utilizando uma escala adaptada de Likert de quatro pontos, contendo seis questões no segundo grupo, nove no terceiro e o último com treze questões. A aplicação dos questionários foi realizada ao longo de 15 dias. Os dados foram tabulados em uma planilha eletrônica visando receber os tratamentos adequados, onde cada variável recebeu uma codificação formada com a letra inicial do grupo e o seu respectivo número, por exemplo, o código D12 corresponde a décima segunda pergunta do grupo "Demanda".

Para o tratamento dos dados coletados, o primeiro grupo "Dados Pessoais" recebeu um tratamento valendo-se das estatísticas univariadas média, desvio padrão e tabela de freqüências. $\mathrm{Na}$ análise dos dados dos três grupos subseqüentes utilizou-se a técnica de regressão logística multinomial e análise fatorial. Como ferramenta computacional, utilizouse o SPSS (Statistical Package for the Social Sciences) versão 14.0.

\subsection{Métodos de análise}

Os métodos de análise utilizados nesta pesquisa foram a regressão logística multinomial e a análise fatorial, sendo estas técnicas consideradas como complementares. $\mathrm{Na}$ aplicação da regressão logística multinomial, buscou-se relacionar as principais características dos grupos "Demanda", "Controle" e "Apoio Social" em relação as suas funções exercidas. $\mathrm{Na}$ análise fatorial, procurou-se identificar os principais fatores que contribuem com o estresse dos indivíduos pesquisados.

\subsection{Regressão logística multinomial}


Ao contrário da regressão logística binária, a multinomial pode ser aplicada quando a variável dependente categórica não é necessariamente dicotômica, ou seja, ela pode assumir outros valores que não somente " 0 " e " 1 ", por exemplo. Para a análise das informações, a regressão logística multinomial internamente agrega o conjunto de dados para formar subpopulações com padrões de covariância idênticos, facilitando os testes preditivos, cálculo de resíduos, e o teste de ajustamento do modelo "goodness-of-fit", que avalia se os valores preditos pelo modelo se adequam aos valores observados (SPSS, 2004; HOSMER; LEMESHOW, 2000).

As premissas básicas a serem atendidas são: a) média condicional da equação de regressão será um valor definido entre "zero" e "um"; b) se os erros da equação seguem a distribuição binária, uma vez que a distribuição binária é válida mesmo para ocorrências de eventos elevados e c) se os resultados obtidos podem ser entendidos na forma de probabilidades (HOSMER; LEMESHOW, 2000). A notação geral adaptada da proposta por Hosmer e Lemeshow (2000) e do SPSS (2004) para o modelo de regressão logística multinomial é descrita tal como se encontra em (1).

$$
\pi_{j}(x)=\frac{e^{g i k}}{e^{g_{i 1}}+e^{g_{i 2}}+\ldots+e^{g_{i k}}}
$$

Onde $\pi_{j}(x)$ representa a probabilidade associada a " $x$ ", o subscrito " $j$ " representa $o$ número de categorias da variável dependente, "g:" é o valor das variáveis explicativas associadas ao evento. Na notação anterior " $\mathrm{g}_{\mathrm{ik}}$ " corresponde a relação matemática definida em (2).

$\mathrm{g}_{\mathrm{k}}=\mathrm{b}_{\mathrm{k} 0}+\mathrm{b}_{\mathrm{k} 1} \mathrm{x}_{\mathrm{i} 1}+\mathrm{b}_{\mathrm{k} 2} \mathrm{x}_{\mathrm{i} 2}+\ldots+\mathrm{b}_{\mathrm{kJ}} \mathrm{x}_{\mathrm{iJ}}$

Onde " $b_{\mathrm{kJ}}$ " é o coeficiente estimador e " $\chi$ " representa as variáveis dependentes da regressão logística multinomial. Na regressão logística multinomial é necessário relacionar cada um dos coeficientes "gk" com as probabilidades de ocorrência pela substituição dos termos na notação geral, tal como pode ser representado em (3).

$$
\pi_{j}(x)=\frac{e^{b_{k 0}+b_{k 1} x_{i 1}+\cdots+b_{k i} x_{i j}}}{e^{b_{10}+b_{11} x_{i 1}+\cdots+b_{1 j} x_{i j}}+\cdots+e^{b_{k 0}+b_{k 1} x_{j 1}+\cdots+b_{k j} x_{i j}}}
$$

Em uma regressão logística multinomial, os valores de "g" são vistos como variáveis contínuas não observáveis, ou seja, não é possível efetuar uma relação linear entre estas variáveis e a variável dependente. Com isso, quando se adiciona uma constante aos valores de "gl", a probabilidade resultante " $\pi_{\mathrm{j}}(\mathrm{x})$ " permanece inalterada. Tendo em vista resolver este problema, o valor de "g." é arbitrariamente considerado como "zero" e a enésima categoria "j" é considerada a categoria de referência que será usada como base para interpretação de todas as demais categorias, fazendo com que internamente todas as demais categorias sejam 


\section{ESTRESSE OCUPACIONAL: INFLUÊNCIA DAS MUDANÇAS GERADAS NA ÁREA DE TECNOLOGIA DURANTE IMPLANTAÇÃO DO SAP R/3}

analisadas em pares em relação a ela e aos moldes da regressão logística binária. Todos os coeficientes são calculados com base no critério interativo de máxima verossimilhança (SPSS, 2004).

\subsection{Análise fatorial}

A estatística descritiva é empregada para caracterização da amostra em análise (MAROCO, 2003). Para tanto, faz uso das seguintes métricas: (a) tabelas de distribuição de freqüência de dados; (b) média aritmética; (c) moda; e (d) desvio padrão, o qual descreve a dispersão da variabilidade dos valores de distribuição da população a partir da média. $\mathrm{O}$ uso da análise fatorial nesta pesquisa apresenta característica descritiva na qual, conforme observam Dillon e Godstein (1984), os resultados obtidos com a aplicação da técnica são interpretados à luz da teoria existente. A análise fatorial tem como principal objetivo o de quantificar "construtos" ou valores não diretamente observáveis (MAROCO, 2003). Para tanto, ela sintetiza as informações de um grande grupo de variáveis em um número menor de variáveis ou fatores, o que simplifica a compreensão dos dados (HAIR JR. et al., 2005).

Por outro lado, um Fator refere-se a uma combinação linear das variáveis originais. Uma das formas de combinação das variáveis é efetuada analisando-se a correlação existente entre elas, onde variáveis altamente correlacionadas são agrupadas juntas, formando um fator independente (DILLON; GOLDSTEIN, 1984). Para Johnson e Wickern (1992, p. 396), na análise fatorial as variáveis são agrupadas em função de suas correlações. Isso significa que as variáveis que compõem um determinado fator devem ser altamente correlacionadas entre si e fracamente correlacionadas com as variáveis que entram na composição do outro fator.

\section{APRESENTAÇÃO E ANÁLISE DOS DADOS}

A estatística descritiva é usada no primeiro grupo do questionário, denominado de "Dados Pessoais", que possui 10 variáveis explanatórias. Cabe ressaltar que, em função dos tratamentos estatísticos efetuados, tal como a identificação dos outliers e, conseqüentemente, eliminação destes da amostra, a quantidade de dados foi alterada, sendo efetivamente menor que os 59 dados originais. Verifica-se que, entre os entrevistados, há maior presença do gênero masculino com $81 \%$. A média de idade estimada é de 31,42 anos com um desvio padrão de 8,08 anos; portanto, a idade dos entrevistados é bastante dispersa. A moda é de 25 anos, identificando assim que o formato da distribuição, comparando-se a moda e a média, é assimétrica. Dos entrevistados, aproximadamente 38\% encontra-se na faixa etária situada entre 25 e 30 anos.

Sobre o estado civil, identificou-se que os casados e solteiros encontram-se na mesma proporção, com $47 \%$ e $48 \%$, respectivamente, enquanto apenas $5 \%$ são separados. No que diz respeito à quantidade de filhos, a maioria dos entrevistados $(60 \%)$ não possui filhos. A residência dos entrevistados é predominantemente na capital Curitiba, com aproximadamente 93\% do total. A escolaridade analisada revela que quase a metade, $46 \%$ dos entrevistados, possui nível superior completo e $31 \%$ já possuem alguma especialização, comprovando alta escolaridade na amostra. Com relação ao tempo de vínculo com a empresa, a média 
encontrada é de 5,77 anos e o valor da moda igual a 1 ano. O cargo de analista de sistemas compõe $38 \%$ da amostra, seguido do suporte com 31\% dos entrevistados. Dos pesquisados, apenas $17 \%$ estão envolvidos com o projeto de implantação do SAP, embora todos os profissionais da área de tecnologia apresentem atividades relacionadas ao novo sistema. $\mathrm{O}$ rendimento salarial dos entrevistados evidencia que a maioria, cerca de $34 \%$, possui renda familiar superior a $\mathrm{R} \$ 4.200,00$ e apenas $2 \%$ possuem renda inferior à $\mathrm{R} \$ 1.200,00$.

Efetuou-se a estimativa do desvio padrão para as perguntas com a maior variação no padrão de resposta (desvio padrão igual à unidade). A variável D16 - Atualmente estou sendo exigido para aprender novas ferramentas tecnológicas do grupo de "Demanda", obteve a média de 2,38 e a variável C25 - A empresa onde atuo apresenta transparência na exposição de novas diretrizes relacionadas à minha área de atuação, do grupo de "Controle", obteve média igual a 2,36. Estes resultados apontam uma freqüência relativa de ocorrência dos eventos. Contudo, o elevado valor do desvio padrão de C25, quando relacionada com a variável Vínculo Empregatício, sugere uma divergência de opiniões entre os profissionais mais antigos e os mais recentes. Ao comparar com outras variáveis, percebe-se que as opiniões são bem diferentes e distribuídas. Os empregados com menos tempo de empresa apresentam 55\% de respostas que indicam transparência da empresa. Dentre os mais antigos, $39,99 \%$ não coadunam com a opinião dos mais novos. Em geral, as contratações mais recentes já explicitam os objetivos compatíveis com o foco organizacional do momento. Os profissionais mais antigos, em geral, já experimentaram situações de mudanças e redirecionamento do foco ao longo dos anos, o que nem sempre é realizado com clareza.

As variáveis D12 - Com que freqüência você tem que trabalhar intensamente (isto é, produzir muito em pouco tempo)? e D13 - Seu trabalho exige demais de você? forneceram os menores valores de desvio padrão, correspondentes a 0,54 e 0,59 respectivamente, mostrando que os entrevistados compartilham da mesma opinião em relação a cada pergunta. As médias obtidas são 1,52 para a variável D12, demonstrando baixa a relativa freqüência, e 1,73 para D13, que pode ser interpretado como relativa freqüência de ocorrência. Ambas revelam uma exigência elevada da organização em relação aos profissionais, o que, conforme o modelo de Exigência-Controle-Suporte de Karasek e Theorell (1990), propicia o surgimento do estresse.

\subsection{Estimação do modelo de regressão logística multinomial}

Visando identificar as características principais dos grupos de funções em relação as variáveis constantes dos grupos Demanda, Controle e Apoio Social empregou-se a técnica de regressão logística multinomial. A variável dependente do modelo é representada pelos Grupos de Funções, com 6 subcategorias distintas: Posição de Liderança, Posição de InfraEstrutura, Posição de Suporte, Consultores, Analista de Sistemas/Desenvolvimento e Funções de Apoio.

O método de estimação usado para seleção do melhor modelo foi o forward stepwise, pelo critério de máxima verossimilhança (Likelihood Ratio - LR). Segundo Hair Jr. et al. (1998), o método stepwise é o processo de estimação de modelos estatísticos onde as variáveis independentes são adicionadas ou retiradas do modelo de acordo com o poder de 


\section{ESTRESSE OCUPACIONAL: INFLUÊNCIA DAS MUDANÇAS GERADAS NA ÁREA DE TECNOLOGIA DURANTE IMPLANTAÇÃO DO SAP R/3}

discriminação que elas agregam ao grupo de variáveis preditivas. Os parâmetros de estimação dos modelos são de 5\% de significância para a entrada das variáveis e $10 \%$ para a saída, desconsiderado a inclusão da constante no modelo. A categoria de referência do modelo estimado é a última categoria da variável dependente "Função de apoio".

O modelo de regressão logística multinomial gerado identificou 5 variáveis consideradas como relevantes estatisticamente, a saber: (a) D15-Trabalho com exigências contraditórias ou discordantes; (b) C19-Trabalho repetitivo; (c) C23-Apresenta conhecimento adequado; (d) C24-Depende de outras pessoas, o que gera desconforto $e$ preocupação; (e) AS34 - Sente-se reconhecido. Dentre as características identificadas pelo modelo, dispostas na Tabela 1, para a classificação dos dados entre os grupos de cargos encontrados, destaca-se a presença das variáveis classificadas no grupo de "Controle" como as mais relevantes, definido por meio da estatística de Wald.

Tabela 1: Estimativa dos Coeficientes “B” da Regressão Logística Multinomial

\begin{tabular}{|c|c|c|c|c|c|c|c|c|c|c|}
\hline \multirow[b]{2}{*}{ : } & \multicolumn{10}{|c|}{ Funções exercidas } \\
\hline & $\begin{array}{c}\text { Posição } \\
\text { Lide- } \\
\text { rança }\end{array}$ & $\begin{array}{c}\text { Wal } \\
\text { d }\end{array}$ & $\begin{array}{l}\text { Posiçã } \\
\text { o } \\
\text { Infra- } \\
\text { Estrut } \\
\text { u-ra }\end{array}$ & Wald & $\begin{array}{l}\text { Posiçã } \\
\text { o } \\
\text { Supor- } \\
\text { te }\end{array}$ & Wald & $\begin{array}{l}\text { Con- } \\
\text { sulto } \\
\text {-res }\end{array}$ & Wald & $\begin{array}{l}\text { Analis- } \\
\text { ta de } \\
\text { Sistem } \\
\text { a/ } \\
\text { Desen- } \\
\text { volvi- } \\
\text { mento }\end{array}$ & Wald \\
\hline D15 & $-0,96$ & 11,03 & 15,46 & 0,001 & $-3,03$ & 102,13 & $-0,25$ & 0,59 & $-2,10$ & 54,41 \\
\hline C19 & $-2,37$ & 20,56 & $-16,02$ & 0 & $-23,53$ & 645,14 & $-2,37$ & 18,33 & $-2,06$ & 19,79 \\
\hline C23 & 3,44 & 7,40 & $-0,46$ & 0 & 26,32 & - & $-2,70$ & 2,74 & 5,29 & 21,16 \\
\hline C24 & 8,04 & 56,89 & $-265,38$ & 0,002 & 6,32 & 36,54 & 8,17 & 57,45 & 8,66 & 67,50 \\
\hline AS34 & $-3,58$ & 37,47 & 79,08 & 0,002 & $-0,09$ & 0,03 & $-1,93$ & 9,26 & $-3,08$ & 30,74 \\
\hline
\end{tabular}

Fonte: Resultados extraídos com base nos dados da pesquisa de campo e processados no SPSS 14.

A escala adotada para todas as variáveis classificadas no grupo de "Demanda" e "Controle" e para as variáveis AS37 e AS38 do grupo de "Apoio Social" indica que os valores menores representam freqüências maiores de ocorrência dos eventos e os valores maiores, menor freqüência de ocorrência. Com isso, o coeficiente " $\mathrm{B}$ " da regressão multinomial (Tabela 1), pode ser interpretado da seguinte forma: quanto maior o valor apresentado, menor a freqüência em que o evento ocorreu e vice-versa. Nas demais variáveis classificadas no grupo Apoio Social, de AS26 até AS36, a codificação efetuada indica a percepção dos pesquisados quanto às características descritas. Com isso, os menores valores representam uma maior percepção dos pesquisados e os maiores valores uma menor percepção. Desta forma, o coeficiente " $B$ " da regressão multinomial é interpretado da seguinte forma: quanto menor seu valor mais fortemente a característica foi percebida pelo pesquisado e vice-versa.

Visando verificar estatisticamente a validade do modelo estimado, é possível utilizar a estatística Wald, onde se rejeita a hipótese nula de que os coeficientes da equação sejam iguais a zero e de que as variáveis independentes selecionadas não produzem efeito sobre a variável dependente, quando o valor de probabilidade calculado é menor do que o nível de 
significância de 0,05. A partir dos resultados obtidos, é possível rejeitar a hipótese nula para todas as categorias analisadas, exceto para a categoria "Posição Infra-Estrutura". Percebe-se que esta última é a posição menos influenciada durante o projeto de implantação do Sistema SAP. Independente do projeto, as funções de infra-estrutura (manutenção de equipamentos de informática) permanecem as mesmas, fator este que contribui para que as variáveis selecionadas pelo modelo, estatisticamente, projetem os resultados do teste Wald observados.

Nesse contexto, percebe-se à luz dos resultados dispostos na Tabela 1 que no caso do grupo de Demanda, a variável que mais se destacou foi a D15 - trabalho com exigências contraditórias ou discordantes. Tal variável foi a que obteve maior impacto em termos estatísticos. A estatística estimada para o teste de hipóteses de Wald, para avaliar a significância dos parâmetros, mostra que as únicas funções exercidas que não sofreram impactos foram as posições infraestrutura e consultores, que obtiveram valores diminutos para esse teste estatístico.

Em relação aos resultados auferidos para a variável C19 - trabalho repetitivo, percebe-se que todas as funções exercidas tiveram um impacto significativo em termos estatísticos, exceto a posição infraestrutura. Contudo, a função exercida que obteve maior impacto foi a posição de suporte, evidenciando que o aumento das rotinas cotidianas pode levar a acomodação e isso traz como conseqüência uma redução do grau de estresse para esta categoria.

Em relação aos resultados obtidos para a variável C23 - apresenta conhecimento adequado, teve impacto significativo para as funções exercidas de posição liderança, posição suporte, consultores e analista de sistemas, sendo esta última função aquela que sofre um maior impacto em termos estatísticos. Neste caso, pode-se evidenciar o fato de que um conhecimento mais adequado, em função daquilo que executa, leva a um aumento do estresse na função desempenhada.

No que tange aos resultados obtidos para a variável C24 - depende de outras pessoas, o que gera desconforto e preocupação, percebe-se que todas as funções exercidas impactaram de forma significativa em termos estatísticos, exceto a posição infraestrutura. Pode-se evidenciar que uma maior dependência de outras pessoas, de modo que gere desconforto e preocupação, leva como conseqüência a um maior estresse àqueles que desempenham a função de analista de sistema/desenvolvimento.

Ao interpretar a variável AS34 - sente-se reconhecido, percebeu-se que todas as estatísticas de Wald referentes às funções exercidas foram estatisticamente significativas, exceto as posições infraestrutura e de suporte que não tiveram significância estatística. Os parâmetros estimados para as posições evidenciam valores negativos e mostram que um menor reconhecimento por parte dos demais colegas leva a um maior grau de estresse.

$\mathrm{Na}$ regressão logística multinomial é possível verificar estatisticamente a contribuição das variáveis selecionadas ao modelo calculado usando-se o teste de "-2 Log Likelihood". Neste teste de hipóteses, o valor "-2 Log Likelihood" representa, para cada variável, um pseudo modelo sem o efeito desta variável e o Qui-quadrado representa a diferença entre o "-2 Log Likelihood" do modelo atual menos o pseudo "-2 Log Likelihood" sem o efeito das variáveis. Para os dados em análise, verifica-se que o valor de probabilidade calculado é inferior ao nível de significância de 0,05 para todas as cinco variáveis selecionadas no 


\section{ESTRESSE OCUPACIONAL: INFLUÊNCIA DAS MUDANÇAS GERADAS NA ÁREA DE TECNOLOGIA DURANTE IMPLANTAÇÃO DO SAP R/3}

modelo. Logo, rejeita-se a hipótese nula de que as variáveis selecionadas não colaboram para o modelo estimado.

Na regressão logística multinomial não é possível aplicar a estatística de ajustamento conhecida como $\mathrm{r}^{2}$, uma vez que não se supõe a normalidade na distribuição dos resíduos. Contudo, existem diversas outras medidas que visam atestar a adequação do modelo e podem substituir a estatística $r^{2}$, pelo chamado de pseudos $r^{2}$. Entre elas, pode-se destacar as estatísticas de Cox \& Snell $r^{2}$, Nagelkerk $r^{2}$ e o teste McFadden. Para interpretação dos resultados obtidos com esses testes é possível verificar que quanto mais próximo de 1 o coeficiente, uma quantidade maior da variação dos dados em análise pode ser explicada por este modelo. Para os dados em análise, verifica-se que o coeficiente do teste de Cox \& Snell $r^{2}$ é igual a 0,916, o coeficiente do teste de Nagelkerk $r^{2}$ é igual a 0,942 e o de McFadden é igual a 0,691 ; ou seja, muito próximos de 1 , significando que entre $69,1 \%$ e $94,2 \%$ das variações podem ser explicadas pelo modelo (SPSS, 2004). Para a efetiva visualização da adequação do modelo aos dados em análise, faz-se necessária a apresentação da matriz de classificação demonstrada na Tabela 2.

Tabela 2: Matriz de Classificação do Modelo de Regressão Logística

\begin{tabular}{|c|c|c|c|c|c|c|c|}
\hline & \multicolumn{7}{|c|}{ Observado } \\
\hline Categorias & $\begin{array}{c}\text { Posição } \\
\text { Lide- } \\
\text { rança }\end{array}$ & $\begin{array}{c}\text { Posição } \\
\text { Infra- } \\
\text { Estru- } \\
\text { tura }\end{array}$ & $\begin{array}{l}\text { Posição } \\
\text { Suporte }\end{array}$ & $\begin{array}{c}\text { Con- } \\
\text { sulto } \\
\text {-res }\end{array}$ & $\begin{array}{c}\text { Analis- } \\
\text { ta } \\
\text { Siste- } \\
\text { mas/ } \\
\text { Desen- } \\
\text { volvi. }\end{array}$ & $\begin{array}{l}\text { Fun- } \\
\text { ções de } \\
\text { Apoio }\end{array}$ & $\begin{array}{c}\% \\
\text { Cor- } \\
\text { reto }\end{array}$ \\
\hline Posição Liderança & 0 & 0 & 0 & 0 & 69 & 0 &, $0 \%$ \\
\hline Posição Infra-Estrutura & 0 & 134 & 0 & 0 & 0 & 0 & $100,0 \%$ \\
\hline Posição Suporte & 0 & 0 & 269 & 0 & 93 & 0 & $74,3 \%$ \\
\hline Consultores & 0 & 0 & 0 & 0 & 33 & 22 &, $0 \%$ \\
\hline $\begin{array}{l}\text { Analista de } \\
\text { Sistemas/Desenvol. }\end{array}$ & 0 & 0 & 55 & 0 & 518 & 0 & $90,4 \%$ \\
\hline Funções de Apoio & 0 & 0 & 0 & 0 & 0 & 70 & $100,0 \%$ \\
\hline Total &, $0 \%$ & $10,6 \%$ & $25,7 \%$ &, $0 \%$ & $56,5 \%$ & $7,3 \%$ & $78,5 \%$ \\
\hline
\end{tabular}

Fonte: Resultados extraídos com base nos dados da pesquisa de campo e processados no SPSS 14.

É possível notar que $78,5 \%$ dos dados em análise pode ser explicado pelo modelo estimado. Também é possível verificar que, para os grupos de funções Posição Liderança e Consultores, os percentuais de acerto foram de $0 \%$, significando que possivelmente outras variáveis não incluídas no modelo, se adicionadas ao mesmo, podem auxiliar na classificação dos dados nestas categorias. É possível verificar, ainda, que o modelo classifica com sucesso os grupos de funções Posição Infra-Estrutura, Analista de Sistemas/Desenvolvimento e Funções de Apoio, gerando um percentual entre 90,4 a 100\% de acerto. O modelo também apresenta excelente resultado quando aplicado aos grupos de funções Posição Suporte, tendo em vista a previsão de acerto de $74,3 \%$. Com isso, pode-se concluir que o conjunto de variáveis no modelo demonstra adequadamente as características comuns aos grupos de funções apresentados pela base de dados em análise. 


\subsection{Identificação dos fatores de estresse}

No emprego da técnica de análise fatorial, o primeiro passo é a construção da matriz de correlação entre as variáveis. Avaliou-se, por meio desta técnica, a confiabilidade da escala de medida. Para isto, os escores para as perguntas individuais que compreendem a escala devem ser correlacionados e, quanto maior forem os coeficientes de correlação, maior será a sua confiabilidade. Para testá-la, pode-se realizar a confiabilidade de coerência interna, usada para avaliar uma escala somada, onde vários itens são somados para formar um escore de um construto. Nesta pesquisa foi utilizado o alfa de cronbach para testar a confiabilidade da escala utilizada. Hair Jr. et al. (2005) explicam que a obtenção do alfa de cronbach se dá por meio da média dos coeficientes de todas as combinações possíveis das metades divididas, o resultado varia entre 0 e 1 . Em pesquisas empíricas considera-se um alfa de cronbach igual a 0,7 como sendo mínimo, embora coeficientes mais baixos possam ser aceitáveis, dependendo do objetivo da pesquisa. O resultado do teste de coerência apresentou um alfa de cronbach de 0,528. O resultado, apesar de ser considerado baixo, não invalida os estudos efetuados, tendo em vista a característica confirmatória pautando-se na teoria abordada.

Os testes KMO (Kaiser-Meyer-Olkin) e de Bartlett indicam a adequação dos dados para a realização da análise fatorial. $\mathrm{O}$ teste $\mathrm{KMO}$ é uma estatística que indica a proporção da variância dos dados que pode ser considerada comum a todas as variáveis, ou seja, que pode ser atribuída a um fator comum, a sua interpretação pode ser vista como: quanto mais próximo de 1 (unidade) melhor o resultado, ou seja, mais adequada é a amostra à aplicação da análise fatorial. O teste de esfericidade de Bartlett procura avaliar se a matriz de correlação é uma matriz identidade, o que indicaria que não há correlação entre os dados. Dessa forma, valendo-se de um nível de significância de $5 \%$, pode-se rejeitar a hipótese nula de que a matriz de correlação é identidade. Tanto o resultado do teste de hipóteses KMO, que obteve um valor de probabilidade igual a 0,638 (maior do que o nível de significância estatística de $0,05)$ quanto o resultado do teste de hipóteses de Bartlett, rejeitaram a hipótese nula e mostram que a base de dados utilizada é adequada à aplicação de análise fatorial.

No quadro 1 é apresentada a classificação dos fatores indicativos do estresse, mensurada a partir da aplicação da técnica de análise fatorial, as variáveis que compõem cada fator e o percentual de variância, que representa o quanto do estresse é explicado para cada fator. A definição de cada fator foi elaborada a partir da compreensão dos significados individuais das variáveis para, posteriormente, associar as variáveis envolvidas em cada fator. Para esta definição, também foi utilizado o modelo de Exigência-Controle-Suporte de Karasek e Theorell (1990). Assim, ao associar as variáveis envolvidas nesta pesquisa, constata-se a existência total desse modelo: Alta Demanda versus Baixo Controle versus

Baixo Apoio Social = Estresse. . Dentre os fatores identificados, observa-se que o fator 1 é o único que denota o envolvimento de todas as variáveis do modelo (Exigência, Controle e Suporte). Para os demais, encontrou-se parte do modelo como Baixo Controle versus Baixo Apoio Social, Alta Demanda versus Baixo Controle, ou ainda Alta Demanda versus Baixo Controle. 
ESTRESSE OCUPACIONAL: INFLUÊNCIA DAS MUDANÇAS GERADAS NA ÁREA DE TECNOLOGIA DURANTE IMPLANTAÇÃO DO SAP R/3

\begin{tabular}{|c|l|}
\hline \multicolumn{2}{|c|}{ Quadro 1 - Fatores indicativos de } \\
\hline FATOR & \begin{tabular}{l}
\multicolumn{1}{c}{ VARIÁVEIS } \\
\hline $\begin{array}{c}\text { Fator 1 } \\
\text { Exigência } \\
\text { Organizacional }\end{array}$
\end{tabular} \\
\cline { 2 - 2 } & $\begin{array}{l}\text { D16-exigência para aprender } \\
\text { novas ferramentas tecnológicas } \\
\text { C17-possibilidade de aprender } \\
\text { coisas novas no trabalho } \\
\text { AS35- recompensas pelos } \\
\text { resultados atingidos } \\
\text { AS36-objetivos da organização em } \\
\text { sintonia com os objetivos } \\
\text { profissionais }\end{array}$ \\
\hline
\end{tabular}

\begin{tabular}{|c|}
\hline $\begin{array}{c}\text { VARIÂNCIA } \\
\text { (\%) }\end{array}$ \\
\hline 26,67 \\
\hline
\end{tabular}

a Análise Fatorial

ANÁLISE

Necessidade do profissional em estar adequado perante as exigências organizacionais. Simultaneamente, há pouco controle sobre as atividades, indicando menor autonomia e maior dependência de outras pessoas/equipes. Há também baixo suporte social. Este fator interfere na recompensa pelos resultados, bem como na sintonia de objetivos entre a organização e o profissional

\begin{tabular}{|c|}
\hline Fator 2 \\
Vida \\
profissional $\mathrm{x}$ \\
pessoal \\
\hline
\end{tabular}

Fator 3

Comunicação organizacional externa e interna

\begin{tabular}{|c|}
\hline Fator 4 \\
Falta de \\
controle \\
\hline
\end{tabular}

Fator 5

Excesso de trabalho

Fator 6

Trabalho em equipe

Fator 7

Objetivo organizacional
C22- cobranças diárias quanto à qualidade excessiva das atividades AS33-vida profissional ocasiona limitações na vida pessoal AS37-cansaço excessivo e desânimo para ir ao trabalho diariamente

AS38-mau humor e irritação diante de fatos antes considerados insignificantes

\section{C25-a empresa apresenta}

transparência na exposição de novas diretrizes relacionadas à área específica de atuação AS26-ambiente calmo e agradável AS30-bom relacionamento com os chefes

C20-escolha sobre COMO fazer o trabalho

C21-escolha sobre O QUE fazer no trabalho

AS34-reconhecimento das demais pessoas da mesma área
D12- intensidade/quantidade de trabalho
D13- exigência do trabalho
C17-possibilidade de aprender coisas novas no trabalho

\section{D14-tempo suficiente para cumprir todas as tarefas \\ C24-dependência de outras pessoas} para conseguir finalizar o trabalho, o que gera desconforto $e$ preocupação

\section{D15-trabalho com exigências contraditórias ou discordantes D16-exigência para aprender novas ferramentas tecnológicas AS28-apoio dos colegas de trabalho}

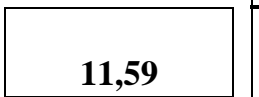

A qualidade no trabalho interfere na disponibilidade e dedicação à vida pessoal, o que se relaciona com a motivação para o trabalho e, conseqüentemente, pode ocasionar alterações de humor e irritabilidade do profissional

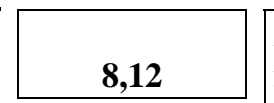

A falta de transparência influencia na qualidade do ambiente de trabalho e no relacionamento com o superior

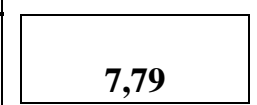

A falta de autonomia interfere no reconhecimento profissional

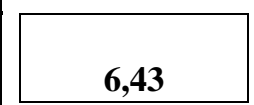

Trabalho excessivo compromete novos aprendizados

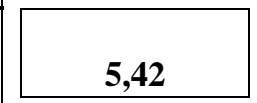

Depender de outras pessoas reflete no cumprimento das tarefas em prazo determinado

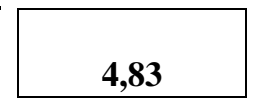

Discordância nas exigências organizacionais influencia na exigência para aprender novas ferramentas e interfere no nível de apoio entre colegas de trabalho

Fonte: Resultados extraídos com base nos dados da pesquisa de campo e processados no SPSS 14. 
Dentre os sete fatores analisados, alguns foram de grande relevância para a constatação do estresse a partir do modelo Exigência-Controle-Suporte de Karasek e Theorell (1990). Os Fatores 1 (exigência organizacional), 4 (falta de controle) e 2 (vida profissional versus vida pessoal) estão devidamente relacionados com o modelo que explica o estresse a partir de elevada exigência versus baixo controle versus falta de apoio social. Todos os demais fatores também apresentam coerência com os preceitos teóricos de Karasek e Theorell, mas os três citados anteriormente são mais puros e propiciam uma compreensão mais imediata. Os Fatores 3 (comunicação organizacional externa e interna) e 7 (objetivo organizacional) demonstram que a organização investigada, no momento da pesquisa, encontrava-se com dificuldades para expor seus objetivos com clareza e coerência. Há, como conseqüência, uma interferência no relacionamento com os pares e os superiores. Estas relações interpessoais também estão sendo afetadas pela dependência de outras pessoas para o cumprimento de tarefas, conforme demonstra o Fator 6 (trabalho em equipe). Os Fatores 2 (vida profissional versus vida pessoal) e 5 (excesso de trabalho) indicam que a organização, no momento da pesquisa, apresentava uma exigência excessiva quanto à qualidade e quantidade de trabalho, o que pode ocasionar restrições na disponibilidade de tempo tanto para novos aprendizados quanto para a dedicação à vida pessoal. Esta última pode ocasionar, ainda, decréscimo da motivação para o trabalho, bem como alterações de humor e irritabilidade.

\section{CONSIDERAÇÕES FINAIS E RECOMENDAÇÕES}

A pesquisa relatada neste artigo teve como objetivo analisar os principais fatores causadores de estresse entre profissionais da área de tecnologia da informação durante um processo de adoção do sistema SAP R/3. O estresse ocupacional é um tema amplamente pesquisado, sobretudo em função dos seus efeitos negativos sobre os resultados organizacionais e sobre a qualidade de vida dos indivíduos, comprovados em diferentes pesquisas. A área de tecnologia, reconhecidamente uma área com nível de estresse elevado, demanda estudos que expliquem as principais causas desse estresse, de forma que possam ser gerenciados de modo efetivo. Em geral, a adoção de novos sistemas gera mudanças significativas em toda a estrutura organizacional, o que aumenta a responsabilidade, por parte dos profissionais de tecnologia, para cumprir prazos e entregar produtos com a menor quantidade de erros.

O estudo de caso foi realizado em uma empresa multinacional instalada em Curitiba, durante o processo de adoção do sistema SAP R/3. Foram identificados os principais fatores estressantes para a amostra intencional de 59 profissionais da área de tecnologia. Com o objetivo de compreender os fatores geradores do estresse, foi aplicado o modelo de Exigência-Controle-Suporte de Karasek e Theorell (1990). A análise dos dados foi quantitativa e realizada em três etapas: (a) descritiva; (b) regressão logística binomial; (c) análise fatorial. A etapa (b) permitiu apreender que o conjunto de variáveis no modelo está adequado às características dos grupos de funções apresentados. Complementarmente, a etapa (c) revelou que existiu a presença de estresse a partir de sete fatores sobressalentes (exigência 


\section{ESTRESSE OCUPACIONAL: INFLUÊNCIA DAS MUDANÇAS GERADAS NA ÁREA DE TECNOLOGIA DURANTE IMPLANTAÇÃO DO SAP R/3}

organizacional, vida profissional $x$ pessoal, comunicação organizacional: externa e interna, falta de controle, excesso de trabalho e objetivo organizacional) e da alta freqüência da combinação: forte exigência, baixo controle e pouco apoio social. Constatou-se, portanto, que o modelo utilizado apresenta compatibilidade para explicar os fatores estressantes entre profissionais de tecnologia, cuja natureza do trabalho envolve excesso de horas trabalhadas, qualidade e rapidez simultaneamente. Esses resultados são relevantes para as organizações que enfrentam situações de mudança decorrentes da adoção de novos sistemas de informação, de modo a reduzir o estresse gerado na equipe de tecnologia com base na gestão adequada dos principais fatores causadores desse estresse.

Para um conhecimento mais aprofundado acerca do estresse entre profissionais de tecnologia, sugere-se a aplicação do mesmo estudo em situações mais estáveis e isentas de fatores externos destoantes dos padrões vigentes na organização, como foi o caso da implementação do SAP. Um estudo comparativo entre filiais pertencentes a uma mesma organização, a fim de identificar eventuais diferenças, também contribuiria para a compreensão dos fatores geradores de estresse. Compreender o quanto a implantação de SAP interfere no estresse dos demais profissionais da empresa, cujas atividades são alteradas em função do novo sistema, contitui outro foco de estudo que pode trazer resultados relevantes para as organizações.

\section{REFERÊNCIAS}

ALBERTIN, A. L. Valor Estratégico dos Projetos de Tecnologia de Informação. Revista de Adminstração de Empresas, v. 41, n. 3, p. 42-50, 2001.

ALVES, M. G. M. et al. Versão Reduzida da "Job Stress Scale": adaptação para o português. Revista Saúde Pública, v. 38, p. 164-171, 2004.

CONTI, R.; ANGELIS, J.; COOPER, C.; FARAGHER, B.; GILL, C. The effects of lean production on worker job stress. International Journal of Operations \& Production Management, v. 26, n. 9, p. 1013-1038, 2006.

DILlON, W. R.; GOLDSTEIN, M. Multivariate Analysis. New York: John Wiley \& Sons, 1984, p. 53-106.

FRANÇA, A. C. L.; RODRIGUES, A. L. Stress e Trabalho: uma abordagem psicossomática. São Paulo: Atlas, 2002.

FOX, S.; STALLWORTH, L. E. The battered apple: An application of stressor-emotioncontrol/support theory to teachers' experience of violence and bullying. Human Relations, v. 63, n. 7, p. 927-954, 2010.

GIL, A. C. Como elaborar projetos de pesquisa. 4. ed. São Paulo: Atlas, 2002.

GILBOA, S.; SHIROM, A.; FRIED, Y.; COOPER, C. A Meta-Analysis of Work Demand Stressors and Job Performance: Examining Main and Moderating Effects. Personnel Psychology, v. 61, p. 227-271, 2008. 
GONÇALVES, J. E. L. As empresas são grandes coleções de processos. Revista de Administração de Empresas, v. 40, n. 1, p. 6-19, 2000.

HAIR Jr. et al. Fundamentos de métodos de pesquisa em administração. Porto Alegre: Bookman, 2005.

HAIR, Jr. et al. Multivariate Data Analysis. $5^{\text {th }}$ Ed. New Jersey: Prentice-Hall, 1998.

HOSMER, D. W.; LEMESHOW, S. Applied logistic regression. $2^{\text {nd }}$ Ed. New York: John Wiley \& Sons, 2000.

JOHNSON, R. A.; WICHERN, D. W. Applied multivariate statistical analysis. 3 ed. New Jersey: Prentice Hall, 1992.

KANJI, G. K.; CHOPRA, P. K. Psychosocial system for work well-being: On measuring work stress by causal pathway. Total Quality Management, v. 20, n. 5, p. 563-580, 2009.

KARASEK, R. A. Job Demands, job decision latitude and mental strain: Implications for job redesign. Administrative Science Quarterly, v. 24, n. 2, p. 285, 1979.

KARASEK, R. A.; THEORELL, T. Healthy work: stress, productivity, and the reconstruction of working life. New York: Basic Books, 1990.

LAKATOS, E. M.; MARCONI, M. A. Fundamentos da metodologia científica. $3^{\text {a }}$. ed. São Paulo: Atlas, 1991, 190p.

LIPP, M.E.N. Stress: conceitos básicos. In: Lipp, M.E.N. (org.). Pesquisas sobre stress no Brasil: saúde, ocupações e grupos de risco. Campinas: Papirus, 1996.

MAROCO, J. Análise Estatística. 2a Ed. Lisboa: Edições Silabo, 2003.

MICHAEL, O.; COURT, D.; PETAL, P. Job stress and organizational commitment among mentoring coordinators. International Journal of Educational Management, v. 23, n. 3, p. 266-288, 2009.

ONGORI, H.; AGOLLA, J. E. Occupational Stress in Organizations and Its Effects on Organizational Performance. Journal of Management Research, v. 8, n. 3, 2008.

RANDALLA, R.; NIELSENB, K.; TVEDTC, S. D. The development of five scales to measure employees' appraisals of organizational-level stress management interventions Work \& Stress, v. 23, n. 1, p. 1-23, 2009.

REVISTA DIGITAL COMPUTERWORLD - 05/2006 “TI é a área mais estressante do mundo, diz pesquisa”. Acesso em 15/07/2006. Disponível em www.computerworld.com.br.

RICHARDSON, K. M.; ROTHSTEIN, H. R. Effects of occupational stress management intervention programs: A meta-analysis. Journal of Occupational Health Psychology, v. 13, n. 1, p. 69-93, 2008.

ROCHA, L. E.; DEBERT-RIBEIRO, M. Trabalho, saúde e gênero: estudo comparativo sobre analistas de sistemas. Revista Saúde Pública, v. 35, n. 6, p. 539-47, 2001. 
ESTRESSE OCUPACIONAL: INFLUÊNCIA DAS MUDANÇAS GERADAS NA ÁREA DE TECNOLOGIA DURANTE IMPLANTAÇÃO DO SAP R/3

SPSS - Statistic Program for Social Science - Tutorial. Release 13.0 ; 01/09/2004.

SELYE, H. Stress of life. Nova York, McGraw-Hill, 1956. 

escala de Likert de 04 pontos

O objetivo deste questionário é coletar dados para trabalharmos com a avaliação do grau de estresse dos trabalhadores desta empresa. Portanto, solicitamos que preencham o questionário com base nas questões a seguir, indicando em que medida cada afirmação está de acordo com o que você pensa, faz ou sente em seu atual momento profissional.

Dados Pessoais

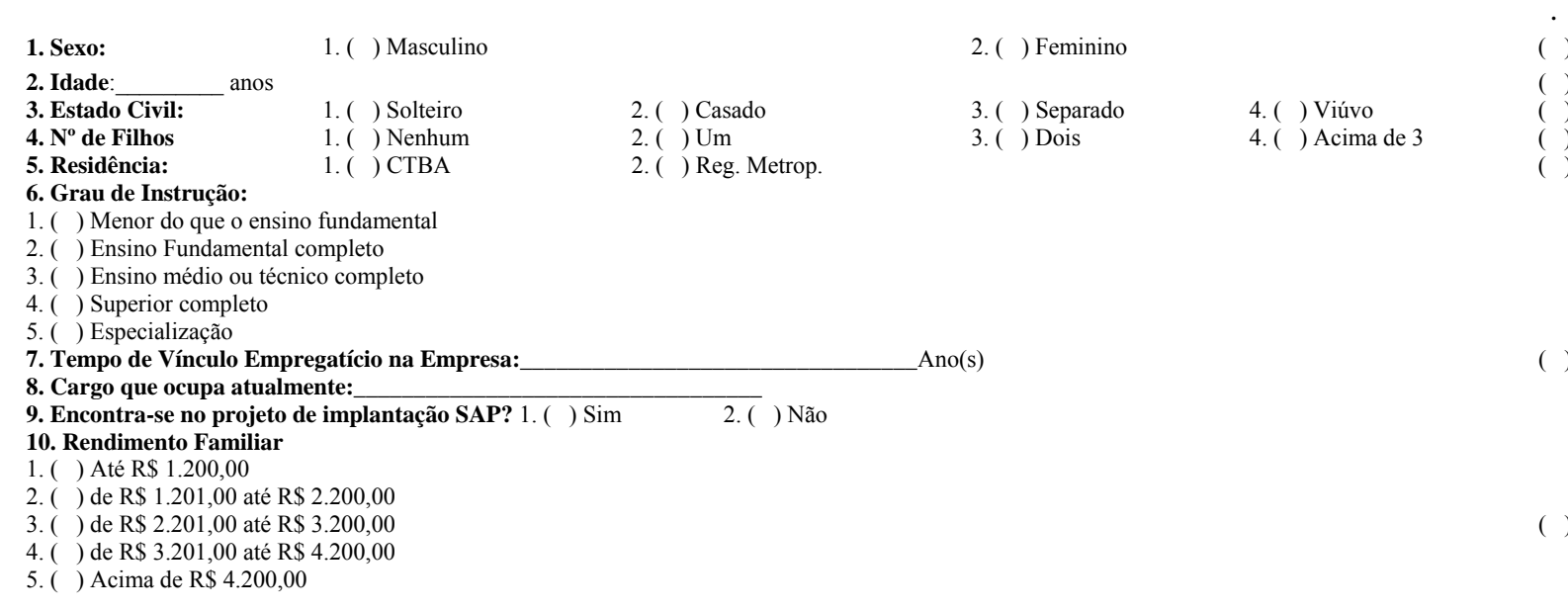

\section{Demanda}

11. Com que frequência você tem que fazer suas tarefas de trabalho com muita rapidez?

$\begin{array}{llll}\text { 1. ( ) Frequentemente } & \text { 2. ( ) As vezes } & \text { 3. ( ) Raramente }\end{array}$

$\begin{array}{lll}\text { 12. Com que freqüência você tem que trabalhar intensamente (isto é, produzir muito em pouco tempo)? } & \text { 2. ( ) As vezes } & \text { 3. ( ) Raramente }\end{array}$

13. Seu trabalho exige demais de você?

$\begin{array}{lll}\text { 1. ( ) Frequentemente } & \text { 2. ( ) As vezes } & 3 .(\text { ) Raramente }\end{array}$

4. ( ) Nunca ou Quase nunca

14. $\mathrm{Voc}$

4. ( ) Nunca ou Quase nunca

4. ( ) Nunca ou Quase nunca

1. ( ) Frequentemente

$\begin{array}{ll}\text { 2. ( ) As vezes } & \text { 3. ( ) Raramente }\end{array}$

15. O seu trabalho costuma apresentar exigências contraditórias ou discordantes?

$\begin{array}{lll}\text { 1. ( ) Frequentemente } & \text { 2. ( ) As vezes } & 3 . \text { ( ) Raramente }\end{array}$

16. Atualmente estou sendo exigido para aprender novas ferramentas tecnológicas.

$\begin{array}{llll}\text { 1. ( ) Frequentemente } & \text { 2. ( ) As vezes } & \text { 3. ( ) Raramente }\end{array}$

4. ( ) Nunca ou Quase nunca

4. ( ) Nunca ou Quase nunca

4. ( ) Nunca ou Quase nunca

Controle

17. Você tem possibilidade de aprender coisas novas em seu trabalho?

1. ( ) Frequentemente
$\begin{array}{ll}\text { 18. Seu trabalho exige muita habilidade ou conhecimentos especializados? }\end{array}$

1. ( ) Frequentemente 2. ( ) As vezes

19. No seu trabalho, você tem que repetir muitas vezes as mesmas tarefas?

$\begin{array}{ll}\text { 1. ( ) Frequentemente } & \text { 2. ( ) As vezes }\end{array}$

20. Você pode escolher COMO fazer o seu trabalho?

$\begin{array}{ll}\text { 1. ( ) Frequentemente } & \text { 2. ( ) As vezes }\end{array}$

21. Você pode escolher $O$ QUE fazer no seu trabalho?

3. ( ) Raramente 4. ( ) Nunca ou Quase nunca

2. ( ) As vezes

3. ( ) Raramente

4. ( ) Nunca ou Quase nunca

3. ( ) Raramente

4. ( ) Nunca ou Quase nunca

22. Diariamente tenho

3. ( ) Raramente

4. ( ) Nunca ou Quase nunca

4. ( ) Nunca ou Quase nunca

1. ( ) Frequentemente

3. ( ) Raramente
tividades.

1. ( Apresento o conh

$\begin{array}{ll}\text { 2. ( ) As vezes } & \text { 3. ( ) Raramente }\end{array}$

4. ( ) Nunca ou Quase nunca

24. Dependo de outras pessoas para conseguir finalizar meu trabalho, o que gera desconforto e preocupação.

1. ( ) Frequentemente $\begin{array}{ll}\text { 2. ( ) As vezes 3. ( ) Raramente } & \end{array}$

4. ( ) Nunca ou Quase nunca

4. ( ) Nunca ou Quase nunca

1. ( ) Frequentemente

2. ( ) As vezes

3. ( ) Raramente

4. ( ) Nunca ou Quase nunca

Apoio Social

26. Existe um ambiente calmo e agradável onde trabalho.

1. ( ) Concordo Fortemente 2. ( ) Concordo mais que discordo

1. ( ) Discordo mais que concordo

27. A empresa onde atuo apresenta transparência na exposição de nova

retrizes relacionadas à minha área

4. ( ) Discordo Totalmente

$\begin{array}{ll}\text { 1. ( ) Concordo Fortemente 2. ( ) Concordo mais que discordo } & \end{array}$ 28. Eu posso contar com o apoio dos meus colegas de trabalho.

$\begin{array}{ll}\text { 1. ( ) Concordo Fortemente } & \text { 2. ( ) Concordo mais que discordo }\end{array}$

1. ( ) Discordo mais que concordo atuação.

1. ( ) Discordo mais que concordo

4. ( ) Discordo Totalmente

29. Se eu não estiver num bom dia, meus colegas compreendem.

$\begin{array}{ll}\text { 1. ( ) Concordo Fortemente 2. ( ) Concordo mais que discordo } & \text { 2 }\end{array}$

1. ( ) Discordo mais que concordo

4. ( ) Discordo Totalmente

30. No trabalho, eu me relaciono bem com meus chefes. 


\section{ESTRESSE OCUPACIONAL: INFLUENCIA DAS MUDANÇAS GERADAS NA ÁREA DE TECNOLOGIA DURANTE IMPLANTAÇÃO DO SAP R/3}

\begin{tabular}{|c|c|c|c|c|}
\hline 1. ( ) Concordo Fortemente & 2. ( ) Concordo mais que discordo & 1. ( ) Discordo mais que concordo & 4. ( ) Discordo Totalmente & ( \\
\hline \multicolumn{5}{|c|}{ 31. Eu gosto de trabalhar com meus colegas. } \\
\hline 1. ( ) Concordo Fortemente & 2. ( ) Concordo mais que discordo & 1. ( ) Discordo mais que concordo & 4. ( ) Discordo Totalmente & \\
\hline \multicolumn{5}{|c|}{ 32. Sinto frustração quanto às decisões tomadas por outras pessoas que afetam ao meu trabalho diretamente. } \\
\hline 1. ( ) Concordo Fortemente & 2. ( ) Concordo mais que discordo & 1. ( ) Discordo mais que concordo & 4. ( ) Discordo Totalmente & \\
\hline \multicolumn{5}{|c|}{ 33. Minha vida profissional ocasiona atualmente limitações em minha vida pessoal. } \\
\hline 1. ( ) Concordo Fortemente & 2. ( ) Concordo mais que discordo & 1. ( ) Discordo mais que concordo & 4. ( ) Discordo Totalmente & \\
\hline \multicolumn{5}{|c|}{ 34. Sinto-me reconhecido pelas pessoas da minha área. } \\
\hline 1. ( ) Concordo Fortemente & 2. ( ) Concordo mais que discordo & 1. ( ) Discordo mais que concordo & 4. ( ) Discordo Totalmente & \\
\hline \multicolumn{5}{|c|}{ 35. Sinto-me recompensado pelos resultados que atinjo a partir de meu trabalho. } \\
\hline 1. ( ) Concordo Fortemente & 2. ( ) Concordo mais que discordo & 1. ( ) Discordo mais que concordo & 4. ( ) Discordo Totalmente & \\
\hline \multicolumn{5}{|c|}{ 36. Os objetivos da organização estão em sintonia com os meus objetivos profissionais. } \\
\hline 1. ( ) Concordo Fortemente & 2. ( ) Concordo mais que discordo & 1. ( ) Discordo mais que concordo & 4. ( ) Discordo Totalmente & \\
\hline \multicolumn{5}{|c|}{ 37. Apresento cansaço excessivo e desânimo para ir ao trabalho diariamente. } \\
\hline 1. ( ) Frequentemente & 2. ( ) As vezes & 3. ( ) Raramente & 4. ( ) Nunca ou Quase nunca & \\
\hline \multicolumn{5}{|c|}{ 38. Em geral, ultimamente tenho apresentado mau humor e irritação diante de fatos que antes considerava insignificantes. } \\
\hline 1. ( ) Frequentemente & 2. ( ) As vezes & 3. ( ) Raramente & 4. ( ) Nunca ou Quase nunca & \\
\hline
\end{tabular}

